\title{
Complex Dynamics in an Evolutionary General Equilibrium Model
}

\author{
Ahmad Naimzada ${ }^{1}$ and Marina Pireddu $\mathbb{D}^{2}$ \\ ${ }^{1}$ Department of Economics, Management and Statistics, University of Milano-Bicocca, U6 Building, \\ Piazza dell'Ateneo Nuovo 1, 20126 Milano, Italy \\ ${ }^{2}$ Department of Mathematics and Its Applications, University of Milano-Bicocca, U5 Building, Via Cozzi 55, 20125 Milano, Italy
}

Correspondence should be addressed to Marina Pireddu; marina.pireddu@unimib.it

Received 29 August 2017; Revised 2 December 2017; Accepted 10 December 2017; Published 16 January 2018

Academic Editor: Ricardo López-Ruiz

Copyright (C) 2018 Ahmad Naimzada and Marina Pireddu. This is an open access article distributed under the Creative Commons Attribution License, which permits unrestricted use, distribution, and reproduction in any medium, provided the original work is properly cited.

\begin{abstract}
We propose an exchange economy evolutionary model with discrete time, in which there are two utility-maximizing groups of agents which differ in the preference structure. Assuming an evolutionary mechanism based on the relative utility values realized by the two kinds of agents, we analytically and numerically investigate the existence of equilibria, their stability, and possible phenomena of coexistence between groups, mainly in terms of the heterogeneity degree in the preference structure. We find that our system has two trivial equilibria, at which just one of the two groups is present, and possibly a nontrivial equilibrium, characterized by the coexistence of the two groups of agents. Such nontrivial equilibrium may be stable, attracting all trajectories, or unstable. In the latter case, interesting, periodic, or chaotic, dynamics arise. We prove that the nontrivial equilibrium emerges via a transcritical bifurcation and loses stability via a flip bifurcation, after which the coexistence between groups is oscillatory in nature, presenting a regular or irregular behavior. In order to better investigate the role of the heterogeneity degree parameter, we perform a bifurcation analysis considering different scenarios, characterized by a balanced or unbalanced endowment distribution of the two goods.
\end{abstract}

\section{Introduction}

Many papers in the economic literature deal with representative agents. Such kind of modeling assumption is justified if the initial heterogeneity among agents asymptotically disappears from the system. In the present work, we show that this is not always guaranteed, by proposing a simple exchange economy evolutionary model with discrete time, in which there are two utility-maximizing groups of agents that differ in the preference structure and where the evolutionary mechanism is based on the relative utility values realized by the two kinds of agents. In such context we more generally investigate, using both analytical and numerical techniques, under which conditions both groups of agents coexist, and thus heterogeneity persists, as well as which are the conditions that lead to the extinction of one of the two groups.

The model we present belongs to the line of research started in [1] and further developed in [2]. Indeed, like in those works, we consider a pure exchange economy model with two utility-maximizing groups of agents heterogeneous in the structure of preferences. Namely, the weights assigned to the two consumption goods in the Cobb-Douglas utility functions do not coincide across groups. Along the paper we shall call the difference between such weights degree of heterogeneity. In $[1,2]$ it was assumed that time is continuous and the focus was on the analysis of the local stability of the equilibria and on some of their static features, such as the coexistence between groups. In those papers no interesting dynamics could arise, because the only possible attractors were given by steady states.

On the other hand, everyday life observation, supported also by empirical data, highlights erratic behaviors in agents' choices, and in particular oscillatory consumption phenomena, such as the fashion cycle (see, e.g., [3, 4]), not reproduced by $[1,2]$. Moreover, from an interpretative viewpoint, the results obtained in the previous works were not suitable to describe the speed with which decisions are taken by real agents. Indeed, in $[1,2]$, the growth rate of each population 
group was determined by a biological payoff function, which depends on the consumption of the group's agents, described in terms of the assumed calorie intakes. In particular, the share updating mechanism is supposed to be monotone in the calorie intakes in [1] and bell-shaped in [2]. According to [1], a monotone population growth rate is suitable to represent the long-run centuries-old trend, as the diet of a population group affects its long-term survival. However, biological payoff functions monotonically increasing in the calorie intakes well describe food regimes characterized by a calorie shortage and thus they are not appropriate to represent the framework of contemporary developed countries and the negative effects of overconsumption on health and survival (see $[2,5]$, and the references therein), which are instead properly described by bell-shaped maps. Hence, the latter setting is suitable to represent the long-run centuries-old trend from the industrial revolution on.

Although the frameworks studied in $[1,2]$ are not wellsuited to describe the quick decisions agents take every day, as observed in the conclusions in [1] the same setup proposed therein can be used to analyze various contexts in which the distribution of preferences changes over time, like, for instance, the evolution of fashion and other trends within a population. Accordingly, in the present work we leave the biological share formation mechanisms considered in the previous papers, replacing them with an economic updating rule. Indeed, inside a pure exchange model in which there are two utility-maximizing groups of agents that differ in the preference structure, we do introduce an evolutionary mechanism based on the relative satisfaction degree, measured as the ratio between functions increasing with the values taken in each time period by the two groups' utility functions, obtaining a framework suitable to describe agents' consumption choices. More precisely, we assume that agents know their own individual satisfaction degree, which coincides with that of the other consumers belonging to the same group and that they are correctly informed about the individual satisfaction degree of the members of the other group, so that they can compare the two values and decide whether to choose again the same preference structure or to switch to the other one. We stress that, different from $[1,2]$, we here suppose that time is discrete rather than continuous. Such choice comes from the consideration that the former framework is more suitable to represent the sequence of actions and decisions which lead to the formation of the population shares. Namely, in view of embracing a new preference structure, agents need to perform the consumption activity, to evaluate the satisfaction degree resulting from their previous choice, to gather information on the other structure of preferences, and to compare the various satisfaction levels, in order to make their next choice. The modeling representation of those actions and decisions requires a time structure which considers an interval between two consecutive time instants. In particular, following [6, 7], we do consider a discrete exponential replicator rule to describe the share updating mechanism. In this context, thanks to the combined effect of the evolutionary mechanism and of the price mechanism, which makes prices move in such a way that demand and supply coincide, we are able to obtain, in addition to static coexistence phenomena between groups, also dynamical coexistence phenomena, oscillatory in nature, in which agents' choices change over time and are not confined just on steady states, describing stationary choices.

Indeed, in such setting, we do investigate from a dynamical viewpoint under which conditions preference heterogeneity implies coexistence of groups characterized by attractors which are not necessarily steady states. More precisely, along the paper, we will try to answer two main questions. The first one is under which conditions one of the two groups asymptotically prevails over the other, leading to the eventual disappearance of the initial agents' heterogeneity. The second one is whether the economy and the population shares set on a stationary equilibrium or on a different (periodic or chaotic) kind of attractor, when groups' heterogeneity asymptotically persists. The answer to the first question is ambiguous, as it depends on the endowment distribution for the two goods. Namely, if the latter is balanced, there exists a nontrivial, that is, characterized by the coexistence between the two groups of agents, equilibrium and agents' heterogeneity persists. If instead the endowment distribution is unbalanced, and thus the endowment values for the two goods differ, a low heterogeneity degree makes agents' heterogeneity disappear. Concerning the answer to the second question, we find that the dynamic nature of the model is influenced by the heterogeneity level, as well as by the endowment distribution. Indeed, if the latter is balanced, the nontrivial equilibrium is stable for low heterogeneity levels, while if the endowment distribution is unbalanced, the nontrivial equilibrium is stable for intermediate heterogeneity degree values. In both frameworks, for larger values of the degree of heterogeneity between groups, the economy and the population shares set on periodic or chaotic attractors.

A paper which bears a resemblance to our work, in regard to the topic and the outcomes, is [8]. Indeed, the common focus is on cultural transmission, for us in the form of transmission of the preference structure due to social imitation, while [8] deals with the problem of cultural transmission of knowledge, habits, and preferences, from parents to their offspring. More precisely, the authors of [8], both in the presence and in the absence of evolutionary selection mechanisms, consider two forms of socialization: the direct vertical one, inside the family, and the oblique one, by society. Parents have to decide how much to invest to affect the preferences of their children, which are also influenced by the number of contacts with the other cultural traits present in the external environment. We observe that we consider just the oblique form of cultural transmission, and not the direct vertical one, but in our model also the price mechanism operates. Concerning the outcomes, [8] analyzes what are the conditions on the transmission mechanisms which induce heterogeneity in the long-run stationary distribution of preferences in the population, while we do also investigate whether the coexistence of different groups can be observed along nonstationary orbits. In particular, we find sufficient conditions for the presence of phenomena of dynamic coexistence which are oscillatory in nature, both regular or not, mainly in terms of the heterogeneity degree. 
We stress that another paper with an approach similar to ours and in which chaotic dynamics are detected, too, is [9], where, however, the authors consider an economy with production and study whether market selection favors profit maximizing firms. We also recall that evolutionary frameworks with binary choices at a collective level have been considered, for instance, in $[10,11]$. On the other hand, different from those papers, the binary choice here occurs between preference structures that characterize the two groups of agents, which are embedded in a general equilibrium framework, where a price mechanism operates.

As we shall see, our assumptions lead us to work with a discrete-time one-dimensional dynamical system, having two trivial equilibria, at which just one of the two groups is present, and possibly a nontrivial equilibrium, characterized by the coexistence between the two groups of agents. According to the considered parameter values, such nontrivial equilibrium may be stable, attracting all trajectories, or unstable. In the latter case, interesting, periodic, or chaotic dynamics arise. We prove that the nontrivial equilibrium emerges via a transcritical bifurcation occurring at one of the two trivial equilibria. As a consequence, when the nontrivial equilibrium emerges, it is locally stable, while the trivial equilibrium it collides with becomes unstable. The nontrivial equilibrium loses stability via a flip bifurcation, after which the coexistence of groups is no more stationary, but oscillatory in nature, presenting a regular or irregular behavior. We stress that a period-doubling bifurcation is indeed the only way in which the nontrivial equilibrium may lose stability, as fold or pitchfork bifurcations would imply the emergence of further nontrivial equilibria, which in our model do not exist. We also analyze the stability of the two trivial stationary equilibria, which amounts to look at the effect produced by their perturbation through the insertion of a share of agents of the missing type. If the considered equilibrium is locally stable, small perturbations will not be successful from an evolutionary viewpoint, being reabsorbed and leading again to the same homogeneous population. On the contrary, insertions of larger shares of agents of the missing type in the case of local stability, or insertions of any size in the case of instability, will not be reabsorbed, leading to a framework characterized just by the presence of the agents of the other group, or to frameworks where the coexistence between the two groups of agents may be stationary or oscillatory, along a periodic or a chaotic trajectory. We find that the stability of the trivial stationary equilibria depends on whether the endowment distribution of the two goods is balanced, and thus the endowment values for the two goods coincide, or not. Moreover, the endowment values influence the stability of the nontrivial equilibrium, too. Due to the central role played by such parameters, in the bifurcation analysis that we perform to better investigate the effect of the heterogeneity degree parameter from a dynamical viewpoint, we consider scenarios characterized just by the features of the endowment distribution of the two goods. In particular, we will study what happens when the distribution is balanced and when it is unbalanced, obtaining the results reported above in answering our two main questions.
The remainder of the paper is organized as follows. In Section 2 we present our model and we find its equilibria, performing some comparative statics exercises, as well as the stability analysis. In Section 3 we further investigate the role of the heterogeneity degree parameter via a bifurcation analysis, considering different scenarios. In Section 4 we briefly discuss our results and describe possible extensions of our model.

\section{The Model}

Let us consider an exchange economy with a continuum of agents, which may be of type $\alpha$ or of type $\beta$. There are two consumption goods, $x$ and $y$, and agent preferences are described by Cobb-Douglas utility functions, that is, $U_{i}(x, y)=x^{i} y^{1-i}$, for $i \in\{\alpha, \beta\}$, with $0<\beta<\alpha<1$. We shall call the parameter $\Delta=\alpha-\beta \in(0,1-\beta)$ degree of heterogeneity between groups. Both kinds of agents have the same endowments of the two goods, denoted, respectively, by $w_{x}$ and $w_{y}$. Time is discrete and the analysis is performed in terms of the relative price $p(t)=p_{y}(t) / p_{x}(t)$, where $p_{x}(t)$ and $p_{y}(t)$ are the prices at period $t$ for goods $x$ and $y$, respectively. We assume that the population is constant at each time period and normalized to 1 , and we denote by $a(t)$ the population fraction composed by the agents of type $\alpha$, so that the population fraction composed by the agents of type $\beta$ is given by $1-a(t)$.

We now present the definition of market equilibrium; we shall refer to in the remainder of the paper.

Definition 1. Given the economy and the population share $a(t)$, a market equilibrium at time $t$ is a vector $\left(p^{*}(t), x_{i}^{*}(t)\right.$, $\left.y_{i}^{*}(t)\right)$, with $i \in\{\alpha, \beta\}$, such that

(i) every kind of agent chooses a utility-maximizing consumption bundle, given $p^{*}(t)$;

(ii) the markets for the two goods are clear.

Simple computations show that, solving the consumer maximization problems for agents of types $\alpha$ and $\beta$ and using a market clearing condition, the market equilibrium price is given by

$$
p^{*}(t)=\frac{[1-(a(t) \alpha+(1-a(t)) \beta)] w_{x}}{(a(t) \alpha+(1-a(t)) \beta) w_{y}}
$$

and the consumer equilibrium quantities of the two goods for an agent of type $i \in\{\alpha, \beta\}$ are

$$
\begin{aligned}
x_{i}^{*}(t) & =i\left(w_{x}+p^{*}(t) w_{y}\right)=\frac{i w_{x}}{a(t) \alpha+(1-a(t)) \beta}, \\
y_{i}^{*}(t) & =(1-i)\left(\frac{w_{x}}{p^{*}(t)}+w_{y}\right) \\
& =\frac{(1-i) w_{y}}{1-(a(t) \alpha+(1-a(t)) \beta)} .
\end{aligned}
$$

See $[1,2]$ for further mathematical details.

Once we specify a dynamical rule for the population share evolution, it is also possible to give the definition of market stationary equilibrium as follows. 
Definition 2. Given the economy, the vector $\left(a^{*}, p^{*}, x_{i}^{*}, y_{i}^{*}\right)$, $i \in\{\alpha, \beta\}$, is a market stationary equilibrium if $a^{*}$ is constant and if given $a^{*},\left(p^{*}, x_{i}^{*}, y_{i}^{*}\right), i \in\{\alpha, \beta\}$, is a market equilibrium for every $t$.

For the sake of brevity, we shall identify market stationary equilibria just with the population share $a$, since it determines all other equilibrium components.

The market stationary equilibria, at which for every $t$ the population shares and thus also the market equilibrium price and the consumer equilibrium quantities are constant, will be called trivial if they are not characterized by the coexistence between the two groups of agents, and nontrivial otherwise.
As concerns the evolutionary mechanism, we assume that agents precisely know their own individual satisfaction degree, which coincides with that of the other consumers belonging to the same group and that they are correctly informed about the individual satisfaction degree of the members of the other group, so that they can compare the two values. The share updating rule is based on such comparison, where the satisfaction degrees are described by the utility values. More precisely, following [6,7], we consider a discrete exponential replicator mechanism, so that the evolution of the fraction $a(t)$ of traders of type $\alpha$ is described by the discrete choice model:

$$
\begin{aligned}
a(t+1) & =\frac{a(t) \exp \left(\mu U_{\alpha}\left(x_{\alpha}^{*}(t), y_{\alpha}^{*}(t)\right)\right)}{a(t) \exp \left(\mu U_{\alpha}\left(x_{\alpha}^{*}(t), y_{\alpha}^{*}(t)\right)\right)+(1-a(t)) \exp \left(\mu U_{\beta}\left(x_{\beta}^{*}(t), y_{\beta}^{*}(t)\right)\right)} \\
& =\frac{a(t)}{a(t)+(1-a(t)) \exp \left(-\mu\left(U_{\alpha}\left(x_{\alpha}^{*}(t), y_{\alpha}^{*}(t)\right)-U_{\beta}\left(x_{\beta}^{*}(t), y_{\beta}^{*}(t)\right)\right)\right)} \\
& =\frac{a(t)}{a(t)+(1-a(t)) \exp \left(-\mu\left(\left(x_{\alpha}^{*}(t)\right)^{\alpha}\left(y_{\alpha}^{*}(t)\right)^{1-\alpha}-\left(x_{\beta}^{*}(t)\right)^{\beta}\left(y_{\beta}^{*}(t)\right)^{1-\beta}\right)\right)},
\end{aligned}
$$

where the positive parameter $\mu$, also called intensity of choice parameter (see [12]), represents the speed of adjustment of the evolutive mechanism and may be interpreted as the population rationality (We stress that the term "rationality" has here to be intended not in the classical sense of the economic analysis, but it has rather to be considered in the context of an imitative decisional mechanism based on a comparison of the utility levels generated by two preference structures. In such framework, for the agents it is "rational" to choose the preference structure that guaranteed the higher utility level in the previous period. See [12, page 133] for a discussion on the topic.) degree. In particular, in the limit $\mu \rightarrow 0$ there is no imitation and (3) simply reads as $a(t+1)=$ $a(t), \forall t$, so that initial conditions about population shares are maintained and agents are insensitive to the relative values of material payoffs. In fact, for $\mu>0$, the right-hand side in (3) represents a monotone increasing function of the difference $U_{\alpha}\left(x_{\alpha}^{*}(t), y_{\alpha}^{*}(t)\right)-U_{\beta}\left(x_{\beta}^{*}(t), y_{\beta}^{*}(t)\right)$. At the other extreme, when $\mu \rightarrow+\infty$, all agents instantaneously move towards the "best" preference structure that guaranteed the higher utility level in the previous time period. If $U_{\alpha}\left(x_{\alpha}^{*}(t), y_{\alpha}^{*}(t)\right)<$ $U_{\beta}\left(x_{\beta}^{*}(t), y_{\beta}^{*}(t)\right)$, then for $\mu \rightarrow+\infty$ we have $a(t+1) \rightarrow 0$, while if $U_{\alpha}\left(x_{\alpha}^{*}(t), y_{\alpha}^{*}(t)\right)>U_{\beta}\left(x_{\beta}^{*}(t), y_{\beta}^{*}(t)\right)$, then $a(t+1) \rightarrow 1$.

We remark that initial conditions about population shares are maintained also when $\alpha=\beta$, that is, when the degree of heterogeneity $\Delta=\alpha-\beta$ is null.

In view of the subsequent analysis, it is expedient to introduce the one-dimensional map $f:[0,1] \rightarrow \mathbb{R}$ related to (3) and defined as

$$
\begin{aligned}
& f(a) \\
& =\frac{a \exp \left(\mu\left(x_{\alpha}^{*}\right)^{\alpha}\left(y_{\alpha}^{*}\right)^{1-\alpha}\right)}{a \exp \left(\mu\left(x_{\alpha}^{*}\right)^{\alpha}\left(y_{\alpha}^{*}\right)^{1-\alpha}\right)+(1-a) \exp \left(\mu\left(x_{\beta}^{*}\right)^{\beta}\left(y_{\beta}^{*}\right)^{1-\beta}\right)} .
\end{aligned}
$$

In the next result, we find the market stationary equilibria for (3).

Proposition 3. Given the economy, the market stationary equilibria for (3) are $a=0, a=1$, and $a=a^{*}$, with

$$
a^{*}=\frac{1-\beta-C \beta}{(\alpha-\beta)(C+1)},
$$

where

$$
C=\left(\frac{(1-\beta)^{1-\beta} \beta^{\beta}}{(1-\alpha)^{1-\alpha} \alpha^{\alpha}}\right)^{1 /(\alpha-\beta)} \frac{w_{y}}{w_{x}}
$$

as long as

$$
\begin{gathered}
\left(\frac{\alpha}{\beta}\right)^{\beta /(\alpha-\beta)}\left(\frac{1-\alpha}{1-\beta}\right)^{(1-\beta) /(\alpha-\beta)}<\frac{w_{y}}{w_{x}} \\
<\left(\frac{\alpha}{\beta}\right)^{\alpha /(\alpha-\beta)}\left(\frac{1-\alpha}{1-\beta}\right)^{(1-\alpha) /(\alpha-\beta)} .
\end{gathered}
$$

Proof. The conclusion immediately follows by observing that $a=0, a=1$, and $a=a^{*}$ in (5) are all the solutions to the fixedpoint equation $f(a)=a$, with $f$ as in (4). The conditions in (7) guarantee that $a^{*} \in(0,1)$. 
We remark that, by construction, at $a=a^{*}$ in (5) it holds that $U_{\alpha}\left(x_{\alpha}^{*}(t), y_{\alpha}^{*}(t)\right)=U_{\beta}\left(x_{\beta}^{*}(t), y_{\beta}^{*}(t)\right)$, that is, at the unique nontrivial equilibrium, characterized by the coexistence of the two kinds of agents, the utility levels generated by the two preference structures coincide.

We shall prove below that for $0<\beta<\alpha<1$ it holds that

$$
\begin{aligned}
& \left(\frac{\alpha}{\beta}\right)^{\beta /(\alpha-\beta)}\left(\frac{1-\alpha}{1-\beta}\right)^{(1-\beta) /(\alpha-\beta)}<1 \\
& <\left(\frac{\alpha}{\beta}\right)^{\alpha /(\alpha-\beta)}\left(\frac{1-\alpha}{1-\beta}\right)^{(1-\alpha) /(\alpha-\beta)}
\end{aligned}
$$

(see Proposition 6). Hence, by (7) and (8), a sufficient condition to have $a^{*} \in(0,1)$ is that $w_{x}$ and $w_{y}$ do not differ too much, that is, that the endowment distribution is balanced. If instead the endowment distribution is strongly unbalanced, then only the trivial equilibria do exist. We shall apply such findings in Section 3.

We stress that even when allowing for heterogeneous endowments of the two goods $x$ and $y$ for the two agents' groups $\alpha$ and $\beta$, that is, even when assuming $w_{x, \alpha} \neq w_{x, \beta}$ and $w_{y, \alpha} \neq w_{y, \beta}$, we would still find, in addition to the two trivial equilibria, a unique nontrivial equilibrium for (3), characterized by the coexistence of the two kinds of agents. For such reason, not to overburden notation and to make computations easier, we decided to confine ourselves to the case of homogeneous endowments.

Before investigating the dynamical features of (3), in the next result, we report some comparative statics exercises on $a^{*}$, which can be derived by direct computations.

Proposition 4. For $a=a^{*}$ in (5) it holds that

$$
\begin{aligned}
& \frac{\partial a^{*}}{\partial w_{x}} \\
& =\frac{\left((1-\beta)^{1-\beta} \beta^{\beta} /\left((1-\alpha)^{1-\alpha} \alpha^{\alpha}\right)\right)^{1 /(\alpha-\beta)} w_{y}}{(\alpha-\beta)\left(\left((1-\beta)^{1-\beta} \beta^{\beta} /\left((1-\alpha)^{1-\alpha} \alpha^{\alpha}\right)\right)^{1 /(\alpha-\beta)} w_{y}+w_{x}\right)^{2}}
\end{aligned}
$$

$>0$,

$$
\begin{aligned}
& \frac{\partial a^{*}}{\partial w_{y}} \\
& =\frac{-\left((1-\beta)^{1-\beta} \beta^{\beta} /\left((1-\alpha)^{1-\alpha} \alpha^{\alpha}\right)\right)^{1 /(\alpha-\beta)} w_{x}}{(\alpha-\beta)\left(\left((1-\beta)^{1-\beta} \beta^{\beta} /\left((1-\alpha)^{1-\alpha} \alpha^{\alpha}\right)\right)^{1 /(\alpha-\beta)} w_{y}+w_{x}\right)^{2}}
\end{aligned}
$$

$<0$.

Hence, according to (9), an increase in $w_{x}$ makes the value of $a=a^{*}$ raise, that is, at the nontrivial equilibrium the share of agents of type $\alpha$ raises when the endowment of good $x$ increases. By (10), an opposite effect on $a=a^{*}$ is obtained by raising $w_{y}$. Such facts may be explained by looking at the expression of the utility functions for the two groups of agents. Indeed, if the population shares were fixed and $w_{x}$ raised, the supply of commodity $x$ would increase, and thus its price would decrease, causing a raise in the equilibrium consumption levels of good $x$ for both groups. Since, however, agents of type $\alpha$ have a stronger preference for commodity $x$ with respect to agents of type $\beta$, the utility level of the former would exceed that of the latter, violating the utility balance condition characterizing $a=a^{*}$, that is, $U_{\alpha}\left(x_{\alpha}^{*}(t), y_{\alpha}^{*}(t)\right)=$ $U_{\beta}\left(x_{\beta}^{*}(t), y_{\beta}^{*}(t)\right)$. Hence, if we want to remain in the steady state $a=a^{*}$, the share of agents of type $\alpha$ has to increase. In this manner, the aggregate demand for commodity $x$ raises and consequently the price of good $x$ tends to increase. Such opposite price effect limits the equilibrium consumption levels of good $x$ for both groups and prevents the utility level of agents of type $\alpha$ from exceeding that of agents of type $\beta$, so that the utility balance condition characterizing $a=a^{*}$ is maintained.

Similarly, if $w_{y}$ raises, the share $1-a^{*}$ of the agents of type $\beta$ has to increase in the nontrivial steady state, leading to a decrease in $a^{*}$, as predicted by (10). Indeed, in such manner, the consequent increased aggregate demand for commodity $y$, which agents of type $\beta$ have a stronger preference for, produces an increase in the price of that good, contrasting the decrease in $p_{y}$ caused by the larger supply of commodity $y$, which, in the absence of a decrease in $a^{*}$, would make the utility level $U_{\beta}\left(x_{\beta}^{*}(t), y_{\beta}^{*}(t)\right)$ exceed $U_{\alpha}\left(x_{\alpha}^{*}(t), y_{\alpha}^{*}(t)\right)$, against the utility balance condition characterizing $a=a^{*}$.

We now turn to the dynamical analysis of (3), by studying in Proposition 5 the local stability of the trivial equilibria and in Proposition 7 the local stability of $a=a^{*}$.

Proposition 5. The equilibrium $a=0$ is locally asymptotically stable under (3) if

$$
\frac{w_{y}}{w_{x}} \in\left(\left(\frac{\alpha}{\beta}\right)^{\alpha /(\alpha-\beta)}\left(\frac{1-\alpha}{1-\beta}\right)^{(1-\alpha) /(\alpha-\beta)},+\infty\right) \text {. }
$$

The equilibrium $a=1$ is locally asymptotically stable under (3) if

$$
\frac{w_{y}}{w_{x}} \in\left(0,\left(\frac{\alpha}{\beta}\right)^{\beta /(\alpha-\beta)}\left(\frac{1-\alpha}{1-\beta}\right)^{(1-\beta) /(\alpha-\beta)}\right) .
$$

Proof. The stability conditions follow by direct computations, imposing, respectively, $-1<f^{\prime}(0)<1$ and $-1<f^{\prime}(1)<1$, with $f$ as in (4). In particular, $-1<f^{\prime}(0)$ and $-1<f^{\prime}(1)$ are always fulfilled, because such derivatives are positive and do not require any restriction on the parameters.

Comparing the stability conditions in Proposition 5 with (7), we notice that when $a^{*}<0$, that is, for $(\alpha / \beta)^{\alpha /(\alpha-\beta)}((1-$ $\alpha) /(1-\beta))^{(1-\alpha) /(\alpha-\beta)}<w_{y} / w_{x}$, then $a=0$ is locally asymptotically stable, while $a=0$ becomes unstable when $a^{*}>0$. As we shall see in Section 3, this happens because, 


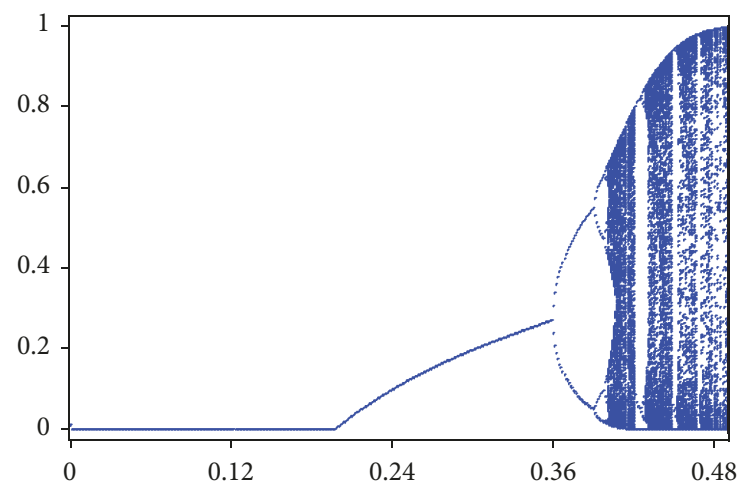

FIgURE 1: The bifurcation diagram of $f$ with respect to $\Delta \in(0,0.49]$, for $w_{x}=1, w_{y}=1.5, a(0)=0.85$.

for suitable parameter values, at 0 a transcritical bifurcation occurs, at which $a=0$ and $a=a^{*}$ merge and the previously stable equilibrium $a=0$ loses its stability in favor of $a=$ $a^{*}$ (see Figure 1). Similar considerations hold for $a^{*}$ in a neighborhood of $a=1$, where a transcritical bifurcation may occur, too (see Figure 3). Hence, the unique nontrivial equilibrium $a=a^{*}$, characterized by the coexistence of the two groups of agents, emerges via a transcritical bifurcation at $a=0$ or at $a=1$. As a consequence, when $a=a^{*}$ enters the interval $(0,1)$, it is stable, while the trivial equilibrium it collides with becomes unstable.

We also notice that, setting

$$
\begin{aligned}
& f_{\beta}(\Delta)=\left(\frac{\beta+\Delta}{\beta}\right)^{(\beta+\Delta) / \Delta}\left(\frac{1-\beta-\Delta}{1-\beta}\right)^{(1-\beta-\Delta) / \Delta}, \\
& g_{\beta}(\Delta)=\left(\frac{\beta+\Delta}{\beta}\right)^{\beta / \Delta}\left(\frac{1-\beta-\Delta}{1-\beta}\right)^{(1-\beta) / \Delta},
\end{aligned}
$$

we may rewrite the stability conditions in Proposition 5 in terms of the heterogeneity degree $\Delta=\alpha-\beta$ as $f_{\beta}(\Delta)<$ $w_{y} / w_{x}$ and $w_{y} / w_{x}<g_{\beta}(\Delta)$, respectively. Moreover, we can equivalently rewrite (8) in terms of $\Delta$ as $F_{\beta}(\Delta)>1>G_{\beta}(\Delta)$, where

$$
\begin{aligned}
F_{\beta}(\Delta) & =\left(f_{\beta}(\Delta)\right)^{\Delta} \\
& =\left(\frac{\beta+\Delta}{\beta}\right)^{\beta+\Delta}\left(\frac{1-\beta-\Delta}{1-\beta}\right)^{1-\beta-\Delta}, \\
G_{\beta}(\Delta) & =\left(g_{\beta}(\Delta)\right)^{\Delta}=\left(\frac{\beta+\Delta}{\beta}\right)^{\beta}\left(\frac{1-\beta-\Delta}{1-\beta}\right)^{1-\beta} .
\end{aligned}
$$

In the next result we show that such reformulation of (8) holds true for $0<\beta<1$ and $\Delta \in(0,1-\beta)$, and we investigate how $f_{\beta}(\Delta)$ and $g_{\beta}(\Delta)$ depend on $\Delta$.
Proposition 6. For $f_{\beta}(\Delta), g_{\beta}(\Delta), F_{\beta}(\Delta)$ and $G_{\beta}(\Delta)$ in (13) and (14), respectively, it holds that

$$
\begin{aligned}
& F_{\beta}(\Delta)>1>G_{\beta}(\Delta), \\
& f_{\beta}^{\prime}(\Delta)>0, \\
& g_{\beta}^{\prime}(\Delta)<0,
\end{aligned}
$$

for $\Delta \in(0,1-\beta)$ and $0<\beta<1$.

Proof. In order to show that $F_{\beta}(\Delta)>1>G_{\beta}(\Delta)$, we observe that

$$
\lim _{\Delta \rightarrow 0^{+}} F_{\beta}(\Delta)=\lim _{\Delta \rightarrow 0^{+}} G_{\beta}(\Delta)=1
$$

Moreover,

$$
F_{\beta}^{\prime}(\Delta)=F_{\beta}(\Delta) \log \left(\frac{(1-\beta)(\beta+\Delta)}{\beta(1-\beta-\Delta)}\right)>0
$$

if and only if $(\beta+\Delta) /(1-\beta-\Delta)>\beta /(1-\beta)$, which is satisfied by $\Delta>0$. Hence, $F_{\beta}^{\prime}(\Delta)>0$ and thus $F_{\beta}(\Delta)>\lim _{\Delta \rightarrow 0^{+}} F_{\beta}(\Delta)=1$, for each $\Delta \in(0,1-\beta), 0<\beta<1$. Furthermore,

$$
\begin{aligned}
G_{\beta}^{\prime}(\Delta) & =\frac{1}{\beta^{\beta}(1-\beta)^{(1-\beta)}}\left(\beta\left(\frac{1-\beta-\Delta}{\beta+\Delta}\right)^{1-\beta}\right. \\
\left.-(1-\beta)\left(\frac{\beta+\Delta}{1-\beta-\Delta}\right)^{\beta}\right) & >0
\end{aligned}
$$

if and only if $\beta /(1-\beta)>(\beta+\Delta) /(1-\beta-\Delta)$, which is fulfilled by $\Delta<0$. Since we do admit just positive values for $\Delta$, then $G_{\beta}^{\prime}(\Delta)<0$ and thus $G_{\beta}(\Delta)<\lim _{\Delta \rightarrow 0^{+}} G_{\beta}(\Delta)=1$, for each $\Delta \in(0,1-\beta)$ and $0<\beta<1$. Thus, $F_{\beta}(\Delta)>1>G_{\beta}(\Delta)$ is fulfilled by any $\Delta \in(0,1-\beta)$ and $0<\beta<1$, as desired.

The fact that $f_{\beta}(\Delta)$ is increasing in $\Delta$ follows by direct computations. Indeed,

$$
\begin{gathered}
f_{\beta}^{\prime}(\Delta)=-\frac{f_{\beta}(\Delta)}{\Delta^{2}}\left(\beta \log \left(\frac{\beta+\Delta}{\beta}\right)\right. \\
\left.+(1-\beta) \log \left(\frac{1-\beta-\Delta}{1-\beta}\right)\right)
\end{gathered}
$$

is positive if and only if

$$
G_{\beta}(\Delta)=\left(\frac{\beta+\Delta}{\beta}\right)^{\beta}\left(\frac{1-\beta-\Delta}{1-\beta}\right)^{1-\beta}<1,
$$

and we proved above that it is true for $\Delta \epsilon(0,1-\beta)$ and $0<\beta<1$. 
Finally, we find the following expression for $g_{\beta}^{\prime}(\Delta)$

$$
\frac{g_{\beta}(\Delta)}{\Delta}\left(\frac{\beta}{\beta+\Delta}-\frac{1-\beta}{1-\beta-\Delta}-\frac{\beta(\log ((\beta+\Delta) / \beta))+(1-\beta)(\log ((1-\beta-\Delta) /(1-\beta)))}{\Delta}\right),
$$

which is negative if and only if

$$
\begin{aligned}
& \exp \left(\frac{\Delta^{2}}{(\beta+\Delta)(1-\beta-\Delta)}\right) \\
& \quad>\left(\frac{\beta}{\beta+\Delta}\right)^{\beta}\left(\frac{1-\beta}{1-\beta-\Delta}\right)^{1-\beta} .
\end{aligned}
$$

In order to show that the latter inequality is fulfilled for $\Delta \epsilon$ $(0,1-\beta)$ and $0<\beta<1$, we observe that, setting

$$
h_{\beta}(\Delta)=\frac{\exp \left(\Delta^{2} /((\beta+\Delta)(1-\beta-\Delta))\right)}{(\beta /(\beta+\Delta))^{\beta}((1-\beta) /(1-\beta-\Delta))^{1-\beta}},
$$

it holds that $\lim _{\Delta \rightarrow 0^{+}} h_{\beta}(\Delta)=1$. If we prove that $h_{\beta}(\Delta)$ is an increasing function, then (22) follows. Indeed, straightforward computations show that $h_{\beta}^{\prime}(\Delta)>0$ if and only if $\Delta^{2}+\beta(1-\beta)>0$, which is true for $\Delta \in(0,1-\beta)$ and $0<\beta<1$. Hence, we have $h_{\beta}^{\prime}(\Delta)>0$, and consequently $g_{\beta}^{\prime}(\Delta)<0$, for $\Delta \in(0,1-\beta)$ and $0<\beta<1$. This concludes the proof.

Hence, increasing $\Delta$ impairs the stability of $a=0$ and of $a=1$, as both intervals in Proposition 5 get reduced. This suggests that $\Delta$ has a destabilizing role: indeed, we shall confirm it in Section 3.

We remark that the trivial stationary equilibria $a=0$ and $a=1$ are homogeneous, in the sense that they are characterized by the presence of a unique kind of agents. Studying their stability means then looking at the effect produced by their perturbation through the insertion of a share of agents of the other type. If the considered equilibrium is locally stable, small perturbations will not be successful from an evolutionary viewpoint, being reabsorbed and leading again to the same homogeneous population. On the contrary, insertions of larger shares of agents of the other type in the case of local stability, or insertions of any size in the case of instability, will not be reabsorbed, leading to a framework characterized just by the presence of the agents of the other group, or to frameworks where the coexistence between the two groups of agents may be stationary or oscillatory in nature, along a periodic or a chaotic trajectory. We will better illustrate the possible different frameworks in Section 3.

In the next result, we focus instead on the stability of the unique nontrivial equilibrium, characterized by the coexistence of the two groups of agents, and we also prove that it loses stability via a flip bifurcation, after which the coexistence is no more stationary, but oscillatory, presenting a regular or irregular behavior. We stress that a perioddoubling bifurcation is indeed the only way in which the nontrivial equilibrium may lose stability, as fold or pitchfork bifurcations would imply the emergence of further nontrivial equilibria, which in our model do not exist.

Due to the analytical difficulties arising when dealing with parameter $\Delta$, in Proposition 7 we focus on the role of the speed of adjustment $\mu$. We shall investigate with the aid of numerical tools the effects produced by the degree of heterogeneity on the stability of $a^{*}$ in Section 3, where we will consider different parameter settings. In particular, we will see that $\Delta$ has a destabilizing effect in all the scenarios and that, increasing its value, the nontrivial equilibrium loses stability again via a flip bifurcation, where the coexistence of groups from stationary becomes oscillatory even in the long run.

Proposition 7. Recalling the definition of $C$ in (6), the equilibrium $a=a^{*}$ in (5) is locally asymptotically stable under (3) if

$$
\begin{aligned}
& \mu<\mu^{*} \\
& =\frac{2 C^{2-\alpha}}{(C+1)(1-\beta-\beta C)(\alpha(C+1)-1)\left(\alpha w_{x}\right)^{\alpha}\left((1-\alpha) w_{y}\right)^{1-\alpha}} .
\end{aligned}
$$

In particular, a flip bifurcation occurs at $a=a^{*}$ for $\mu=\mu^{*}$.

Proof. The stability condition follows by imposing $1>$ $f^{\prime}\left(a^{*}\right)>-1$, with $f$ as in (4). Actually, $1>f^{\prime}\left(a^{*}\right)$ is always fulfilled and does not require any restriction on the parameters. Direct computations show that the factors (1 $\beta-\beta C)$ and $(\alpha(C+1)-1)$ are positive if and only if $(7)$ is satisfied, that is, when $a^{*} \in(0,1)$. The condition for the flip bifurcation follows by setting $f^{\prime}\left(a^{*}\right)=-1$.

Although in Section 3 we will mainly rely on numerical simulations to investigate the role of $\Delta$, we stress that as we will more precisely explain in the next section, it is possible to use the stability condition for $a=a^{*}$ obtained in Proposition 7 to double-check the stability threshold value of $\Delta$ that we derive in Proposition 9 and those we infer from the bifurcation diagrams reported in Figures 3 and 5.

Still in regard to local stability, we finally remark that also the endowment values asymptotically affect the system behavior. Indeed, this is evident from the stability conditions found in Proposition 5 for $a=0$ and $a=1$. Moreover, we observe that $w_{x}$ and $w_{y}$ influence the stability of $a=a^{*}$, too. In fact, the stability condition derived in Proposition 7 for $a=a^{*}$, when expanded, contains terms of degree 3 in $w_{x}$ and $w_{y}$. Finding the stability threshold values in terms of such parameters would then require cumbersome computations. Nonetheless, due to the central role they play, the scenarios we will consider in Section 3 are characterized just by the features of the endowment distribution of the two goods. In particular, we will investigate what happens when it is balanced, that is, $w_{x}=w_{y}$, and when it is unbalanced. 


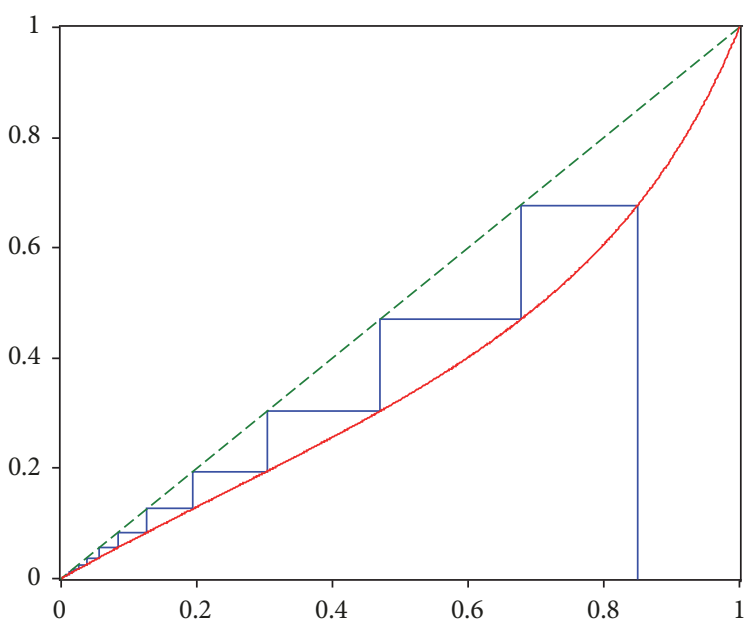

(a)

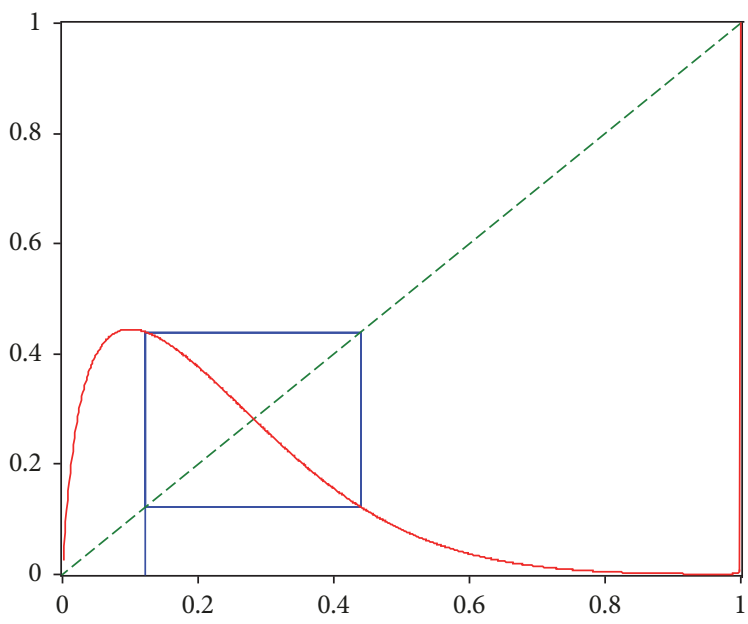

(c)

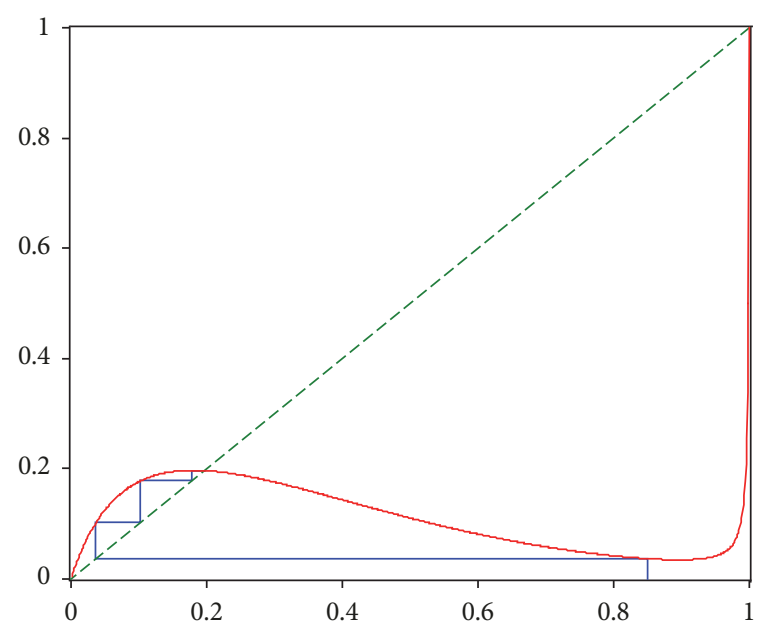

(b)

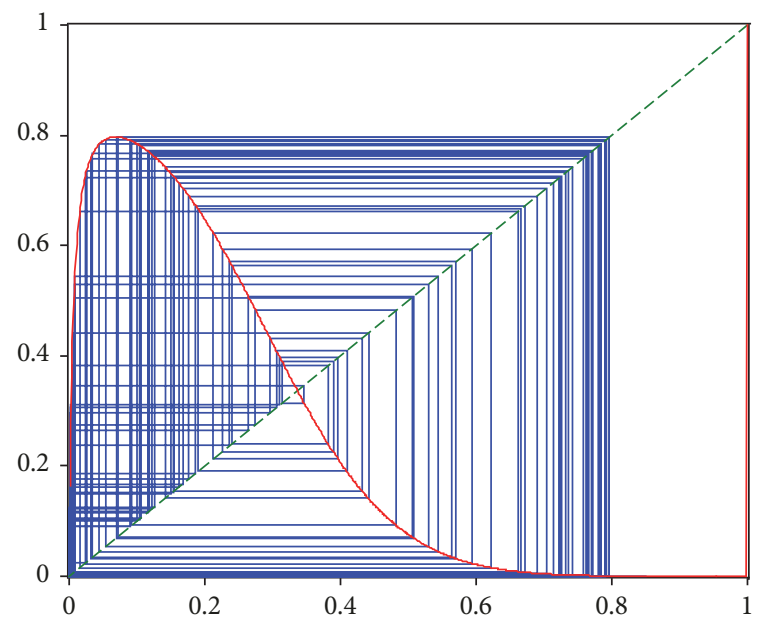

(d)

FIGURE 2: The graph of $f$ for $w_{x}=1, w_{y}=1.5$, and $\Delta=0.1, a(0)=0.85$ in (a), $\Delta=0.3, a(0)=0.85$ in (b), $\Delta=0.37, a(0)=0.122$ in $(\mathrm{c})$ and $\Delta=0.42, a(0)=0.85$ in (d), respectively.

Let us conclude the present section by summarizing the possible dynamical behaviors displayed by our model. When $a^{*} \notin(0,1)$, then $a=0$ may be unstable and $a=1$ stable, or vice versa. Hence, in such setting, according to the considered parameter values, one of the groups totally prevails over the other and preference heterogeneity disappears. When instead $a^{*} \in(0,1)$, it may be locally stable or unstable. In the former case, since $a^{*}$ is characterized by the coexistence between the two groups of agents, we have that preference heterogeneity persists, being not absorbed by the market and evolutive mechanisms. This happens also when $a^{*} \in(0,1)$ but it is unstable, the only difference with the previous framework being the oscillatory, periodic, or chaotic behavior of the system. For an illustration of the above described scenarios, see Figures 2, 4, and 6.

\section{The Role of the Heterogeneity Degree}

With the aid of our simple evolutionary general equilibrium model we are interested in answering two main questions.

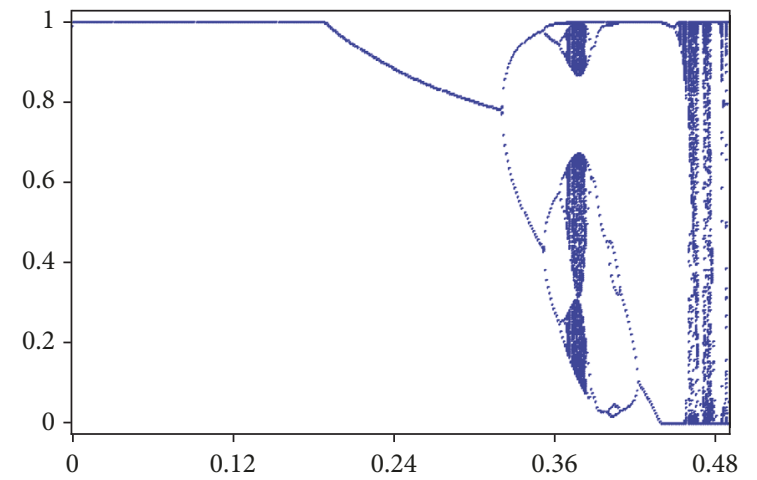

FIGURE 3: The bifurcation diagram of $f$ with respect to $\Delta \in(0,0.49]$, for $w_{x}=1.5, w_{y}=1, a(0)=0.15$.

The first one is under which conditions one of the two groups of agents asymptotically prevails over the other, leading to the eventual disappearance of the initial population heterogeneity, while the second one is whether the economy 


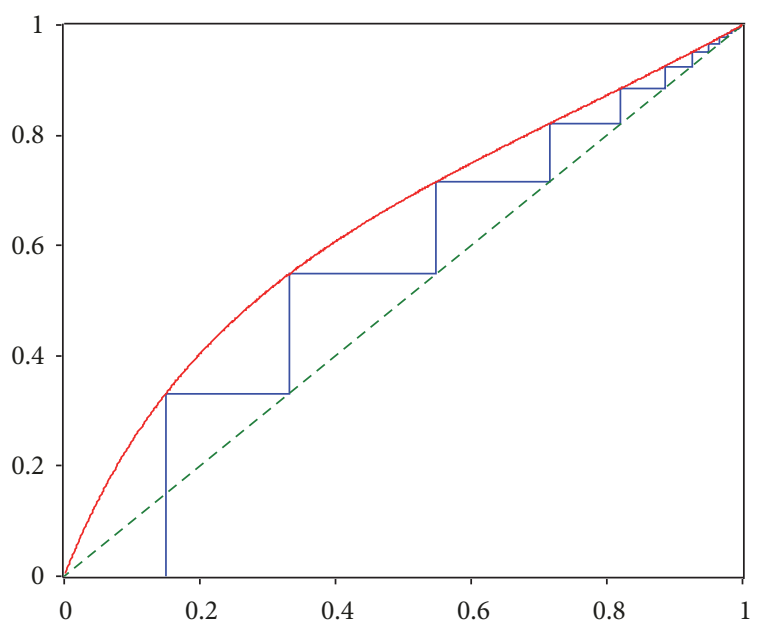

(a)

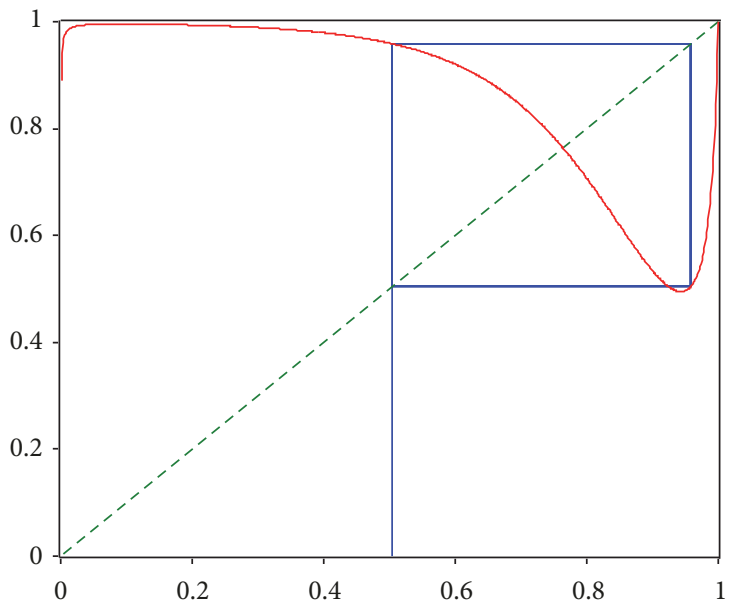

(c)

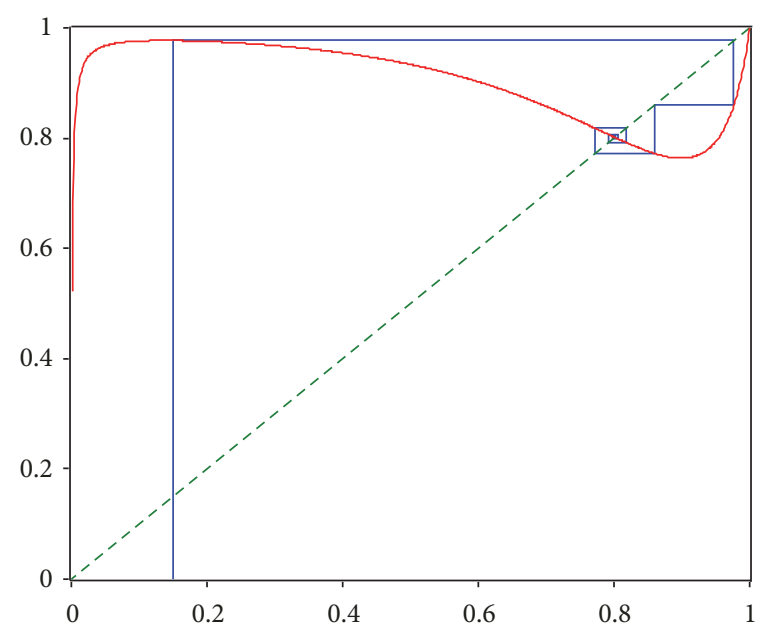

(b)

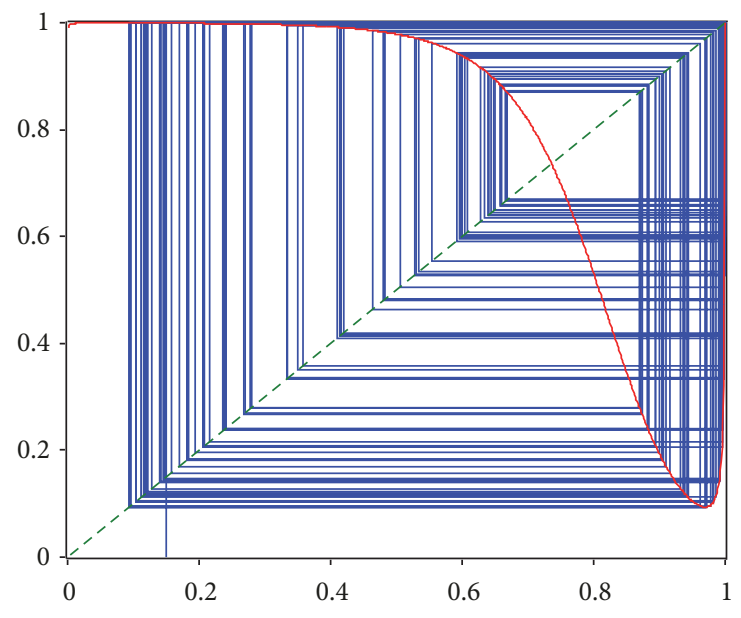

(d)

Figure 4: The graph of $f$ for $w_{x}=1.5, w_{y}=1$, and $\Delta=0.1, a(0)=0.15$ in (a), $\Delta=0.3, a(0)=0.15$ in (b), $\Delta=0.34, a(0)=0.505$ in $(\mathrm{c})$ and $\Delta=0.38, a(0)=0.15$ in $(\mathrm{d})$, respectively.

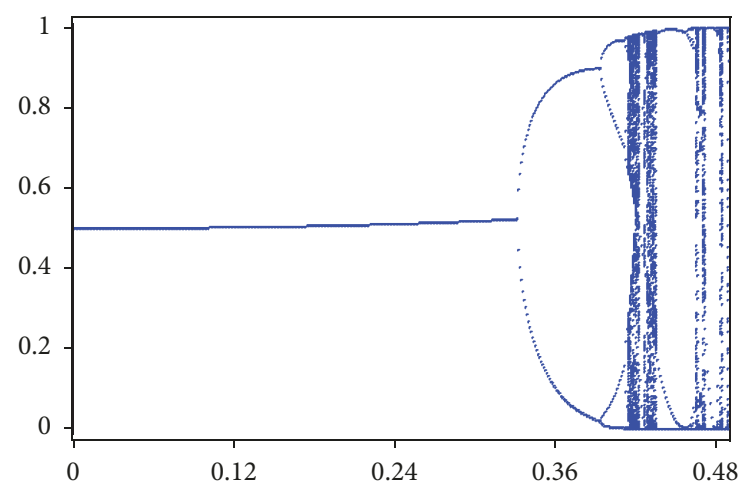

Figure 5: The bifurcation diagram of $f$ with respect to $\Delta \in(0,0.49]$, for $w_{x}=w_{y}=1, a(0)=0.5$.

and the population shares set on a stationary equilibrium or on a different (periodic or chaotic) kind of attractor, when groups' heterogeneity asymptotically persists. As we shall see, the answer to both questions depends on whether the endowment distribution for the two goods is balanced or not and on the heterogeneity degree level.

In particular, in Section 2, we observed that, when the degree of heterogeneity $\Delta=\alpha-\beta$ is null, the map $f$ in (4) simply reads as $f(x)=x$ and initial conditions about population shares are maintained. As $\Delta$ slightly increases, we find a regime characterized by the presence of a unique globally asymptotically stable equilibrium which, according to the considered parameter configuration, may be given by $a=0$, like in Figure 1, by $a=1$, like in Figure 3, or by $a=a^{*} \in(0,1)$, like in Figure 5. We will analyze what happens when stability is lost in the bifurcation analysis below.

We stress that, as mentioned in Section 2, when starting from a trivial equilibrium, characterized by a homogeneous population, considering initial conditions about population shares coincides with studying that equilibrium stability, which in turn amounts to investigate whether, asymptotically, the same homogeneous population regime persists, or whether, on the contrary, a different homogeneous population regime or an heterogeneous population regime does 


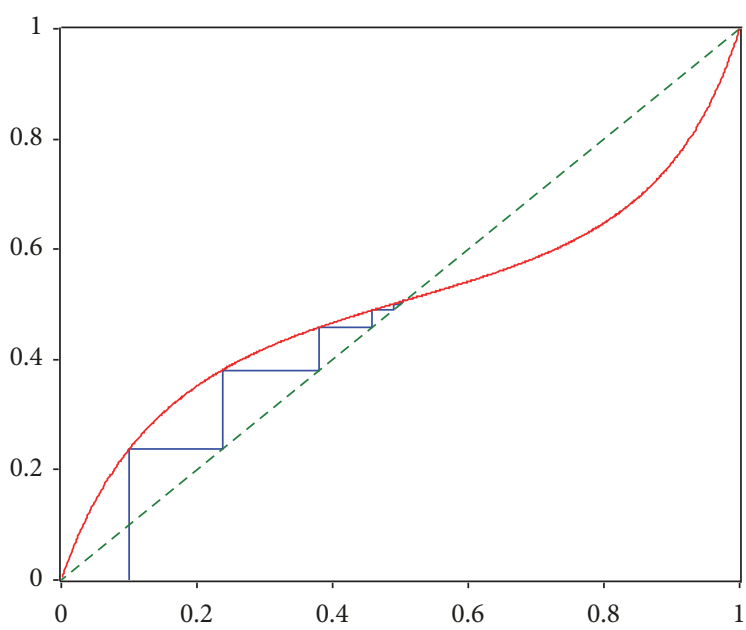

(a)

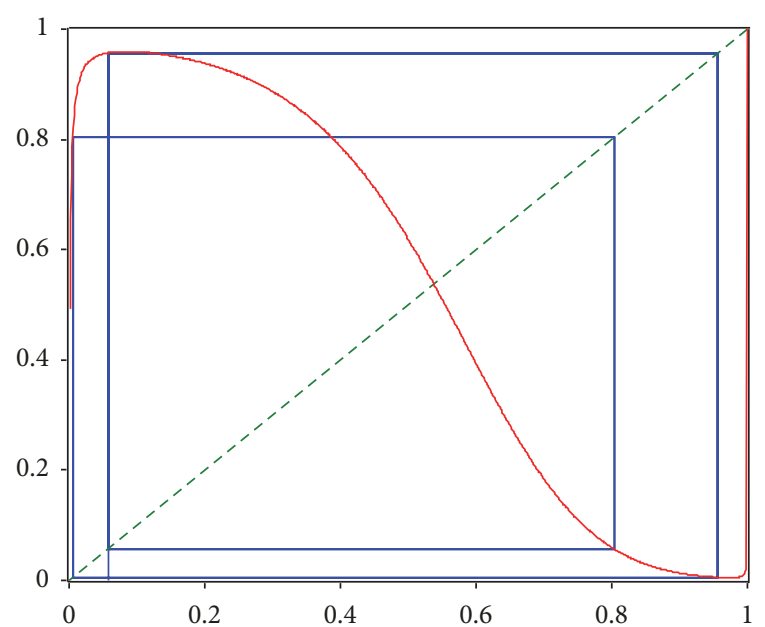

(b)

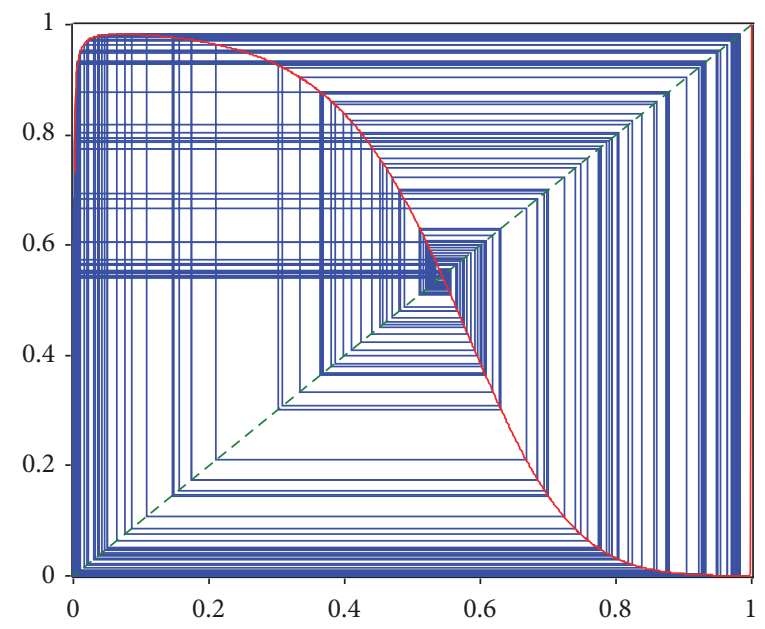

(c)

Figure 6: The graph of $f$ for $w_{x}=w_{y}=1$, and $\Delta=0.2, a(0)=0.1$ in (a), $\Delta=0.40, a(0)=0.058$ in (b) and $\Delta=0.42, a(0)=0.1$ in (c), respectively.

prevail. Indeed, when starting from a trivial equilibrium, the initial condition $a(0) \in(0,1)$ can be seen as a shock, that is, as a perturbation of the uniform population distribution through the insertion of a share of agents of the other group. If the considered trivial equilibrium is locally stable, small perturbations will not be successful from an evolutionary viewpoint, being reabsorbed and leading again to the same homogeneous population. This happens, for instance, in Figure 2(a) with the equilibrium $a=0$, which, however, is globally asymptotically stable. In such case, every shock $a(0) \in(0,1)$ is reabsorbed and eventually the agents of type $\alpha$ disappear from the economy. On the other hand, if the considered trivial equilibrium is unstable, insertions of any share of agents of the other type are not reabsorbed, leading to a framework characterized just by the presence of the other group's agents (like in Figure 4(a) when starting from $a=0$ ), or to frameworks where the coexistence between the two groups of agents may be stationary (like in Figures 2(b) and $4(\mathrm{~b})$ ) or oscillatory in nature, both regular (see Figures 2(c) and 4(c)) or not (see Figures 2(d) and 4(d)).
3.1. Unbalanced Endowment Distribution. At first, we focus on the framework in which the endowment distribution for the two goods is unbalanced, that is, $w_{x} \neq w_{y}$. In particular, in Figure 1, we consider the following parameter setting: $w_{x}=1$, $w_{y}=1.5, \beta=0.5, \mu=15$ and $\Delta$ varies in $(0,0.49]$. For $\Delta$ close to 0 , the stationary equilibrium $a=a^{*}$ in (5) lies outside $(0,1)$ as the right inequality in (7) is not satisfied, and it enters the unit interval through a transcritical bifurcation occurring at $a=0$ for $\Delta=\widetilde{\Delta}=0.197$, when $a=0$ loses stability in favor of $a=a^{*}$. After the transcritical bifurcation, the stable equilibrium is no more the homogeneous $a=0$, characterized by the presence of agents of type $\beta$ only, but $a=a^{*}$, at which the two groups coexist. For $\Delta=\widehat{\Delta}=0.360$, $a=a^{*}$ undergoes a flip bifurcation. In Figure 2 we show the graph of $f$, as well as its forward iterates, for different values of $\Delta$. For $\Delta=0.1$ in (a) we observe a converging behavior towards $a=0$, for $\Delta=0.3$ in (b) trajectories tend to $a=a^{*}$, for $\Delta=0.37$ in (c) we find a period-two cycle and for $\Delta=0.42$ in (d) dynamics become complex due to the presence of a chaotic attractor. 
We state in a formal manner the results about the transcritical and the flip bifurcations, respectively, in Propositions 8 and 9 below (We remark that the occurrence of the transcritical and period-doubling bifurcations we observe in Figures 3 and 5 could be proven as done in Propositions 8 and 9. However, in order not to overburden the paper, we chose to omit such results. We also stress that the value for $\Delta$ we find in Proposition 9 in correspondence to the flip bifurcation is consistent with the value of $\mu^{*}$ derived in Proposition 7: namely, when inserting $\Delta=0.360$ in that expression, together with the other parameter values considered in this scenario, we obtain $\mu^{*}=15$, which coincides with the value for $\mu$ we are taking into account. The same comment applies also to the period-doubling bifurcations occurring in Figures 3 and 5.).

Proposition 8. For the map $f=f(a ; \Delta)$ in (4) a transcritical bifurcation occurs at $\tilde{a}=0$ for $\widetilde{\Delta}=0.197$.

Proof. According to [13, page 507], for the occurrence of a transcritical bifurcation at a point $a=\tilde{a}$ for a certain $\Delta=\widetilde{\Delta}$ we just have to check the following conditions:

$$
\begin{aligned}
f(\tilde{a} ; \tilde{\Delta}) & =\tilde{a}, \\
\frac{\partial f}{\partial a}(\tilde{a} ; \tilde{\Delta}) & =1, \\
\frac{\partial f}{\partial \Delta}(\tilde{a} ; \tilde{\Delta}) & =0, \\
\frac{\partial^{2} f}{\partial a \partial \Delta}(\tilde{a} ; \tilde{\Delta}) & \neq 0, \\
\frac{\partial^{2} f}{\partial a^{2}}(\tilde{a} ; \tilde{\Delta}) & \neq 0 .
\end{aligned}
$$

Direct (software-assisted) computations show that the above conditions are satisfied at $\tilde{a}=0$ for $\widetilde{\Delta}=0.197$. In particular, it holds that $\left(\partial^{2} f /(\partial a \partial \Delta)\right)(\widetilde{a} ; \widetilde{\Delta})=7.879$ and $\left(\partial^{2} f / \partial a^{2}\right)(\widetilde{a} ; \widetilde{\Delta})=$ -5.719 . This completes the proof.

Proposition 9. For the map $f=f(a ; \Delta)$ in (4) a perioddoubling bifurcation occurs at $\widehat{a}=a^{*}=0.271$ for $\widehat{\Delta}=0.360$.

Proof. According to [13, page 516], for the occurrence of a period-doubling bifurcation at a point $a=\hat{a}$ for a certain $\Delta=\widehat{\Delta}$ we have to check the following conditions:

$$
\begin{aligned}
f(\widehat{a} ; \widehat{\Delta}) & =\widehat{a}, \\
\frac{\partial f}{\partial a}(\widehat{a} ; \widehat{\Delta}) & =-1, \\
\frac{\partial f^{2}}{\partial \Delta}(\widehat{a} ; \widehat{\Delta}) & =0, \\
\frac{\partial^{2} f^{2}}{\partial a \partial \Delta}(\widehat{a} ; \widehat{\Delta}) & \neq 0, \\
\frac{\partial^{2} f^{2}}{\partial a^{2}}(\widehat{a} ; \widehat{\Delta}) & =0, \\
\frac{\partial^{3} f^{2}}{\partial a^{3}}(\widehat{a} ; \widehat{\Delta}) & \neq 0 .
\end{aligned}
$$

Direct (software-assisted) computations show that the above conditions are satisfied at $\widehat{a}=0.271$ for $\widehat{\Delta}=0.360$. In particular, it holds that $\left(\partial^{2} f^{2} /(\partial a \partial \Delta)\right)(\widehat{a} ; \widehat{\Delta})=35.937$ and $\left(\partial^{3} f^{2} / \partial a^{3}\right)(\widehat{a} ; \widehat{\Delta})=-73.518$. This completes the proof.

In Figure 3 we consider a different unbalanced endowment distribution setting, fixing all the parameters as in Figure 1, except for $w_{x}=1.5$ and $w_{y}=1$, so that now the endowment of good $x$ exceeds that of good $y$. Also in this case for $\Delta$ close to 0 the stationary equilibrium $a=a^{*}$ in (5) lies outside $(0,1)$ as the left inequality in (7) is not satisfied, it enters the unit interval through a transcritical bifurcation at $a=1$ for $\Delta=0.188$ and then undergoes a flip bifurcation for $\Delta=0.321$. In Figure 4 we show the graph of $f$, as well as its forward iterates, for different values of $\Delta$. For $\Delta=0.1$ in (a) we observe a converging behavior towards $a=1$, for $\Delta=0.3$ in (b) trajectories tend to $a=a^{*}$, for $\Delta=0.34$ in (c) we find a period-two cycle and for $\Delta=0.38$ in (d) dynamics become complex due to the presence of a chaotic attractor.

Comparing the two frameworks above, we notice that switching the values for $w_{x}$ and $w_{y}$ produces a nearly symmetric behavior for $f$, concentrated in the second setting around $a=1$ rather than around $a=0$. Nonetheless, in both settings we found that for low values of $\Delta$ the only market stationary equilibria are $a=0$ and $a=1$, one of which is stable and the other one unstable. Increasing $\Delta$ produces, through a transcritical bifurcation, the emergence of an internal stable equilibrium $a=a^{*}$ from the side of the previously stable equilibrium, so that the extremal equilibria become unstable. Raising $\Delta$ further, also the internal equilibrium loses stability via a flip bifurcation and undergoes a cascade of perioddoubling bifurcations leading to chaos. However, while in Figure 1 we just observe an increasing complexity with the raise of $\Delta$, in Figure 3 we find a phenomenon of "bubbling" (see [14-18]) between the first and the last period-two cycles. Indeed, in such region $a=a^{*}$ is always unstable, but with the increase of $\Delta$ the system complexity grows until a four-piece chaotic attractor and then decreases until the last period-two cycle, which undergoes a flip bifurcation leading to a onepiece chaotic attractor.

Looking at Figures 1 and 3, we notice that between the transcritical and the flip bifurcations the stable equilibrium is given by $a=a^{*}$, characterized by the coexistence of the two groups of agents. This means that it is not true that the group with a stronger preference for the more abundant good necessarily prevails, because of agents' pressure on the price formation mechanism. Indeed, even if the endowment of a commodity is larger with respect to the endowment of the other good, its price raises if the preferences for that good become excessive and the demand for it is too high. Due to the increased price, the agents who prefer it start consuming also the other commodity and this induces a decrease in their payoff, so that the share of the agents belonging to the other group grows and in such way no group disappears from the economy. When, however, the demand for a good is too high, its price increases too much and the agents who have a stronger preference for that good demand consume just a little of that commodity. This produces a reduction of their share and the weak aggregate demand for that good makes 
its price decrease and the demand raises again. In such way, it is possible to have, for instance, a period-two cycle after the flip bifurcation. We stress that a similar argument justifies the existence of periodic orbits also in the case in which the endowments for the two goods coincide, as considered below and illustrated in Figure 5.

3.2. Balanced Endowment Distribution. We now focus on the balanced endowment distribution setting, in which $w_{x}=w_{y}$, considering in Figure 5 all the parameters as in Figure 1, except for $w_{x}=w_{y}=1$. In this case for $\Delta$ close to 0 the stationary equilibrium $a=a^{*}$ in (5) already belongs to $(0,1)$ and it is stable, it undergoes a flip bifurcation for $\Delta=0.332$, followed by a period-doubling cascade leading to a regime characterized by the presence of chaotic regions alternating with periodicity windows.

In such framework, no transcritical bifurcations occur, and increasing $\Delta$ has just a destabilizing effect on $a^{*}$. In Figure 6 we show the graph of $f$, as well as its forward iterates, for different values of $\Delta$. For $\Delta=0.2$ in (a) trajectories tend to $a=a^{*}$, for $\Delta=0.40$ in (b) we find a period-four cycle and for $\Delta=0.42$ in (c) dynamics become complex due to the presence of a chaotic attractor.

Comparing the results obtained in Sections 3.1 and 3.2, we find that the answer to our first main question, that is, under which conditions one of the two groups asymptotically prevails over the other, depends for us on the endowment distribution for the two goods. Indeed, if the latter is balanced, there exists a nontrivial equilibrium, characterized by the coexistence of the two groups of agents, and agents' heterogeneity persists. If instead the endowment distribution is unbalanced, a low heterogeneity degree makes agents' heterogeneity disappear.

As concerns the answer to the second main question, that is, whether the economy and the population shares set on a stationary equilibrium or on a different (periodic or chaotic) kind of attractor, when groups' heterogeneity asymptotically persists, we find that if the endowment distribution is balanced, the nontrivial equilibrium is stable for low heterogeneity levels, while if the endowment distribution is unbalanced, the nontrivial equilibrium is stable for intermediate heterogeneity degrees. In both frameworks, for larger values of the heterogeneity level, the economy sets on periodic or chaotic attractors.

\section{Conclusion}

In the present paper we considered a pure exchange economy model with two utility-maximizing groups of agents heterogeneous in the structure of preferences over two consumption goods. Introducing an evolutionary monotone selection mechanism based on relative agents' material payoffs, we answered two main questions. The first one is under which conditions one of the two groups asymptotically prevail over the other, leading to the eventual disappearance of the initial agents' heterogeneity, while the second one is whether the economy and the population shares set on a stationary equilibrium or on a different (periodic or chaotic) kind of attractor, when groups' heterogeneity asymptotically persists.
We found that the answers to both questions depend on the balance degree of the endowment distribution for the two goods, as well as on the heterogeneity level between groups, which influences the dynamic nature of the model.

We stress that our study has been stimulated by the observation that many papers in the economic literature deal with representative agents and that such kind of modeling assumption is justified if agents' heterogeneity asymptotically disappears from the system. In our model, we showed that this is not always guaranteed. In particular, different from $[1,2]$, we assumed that time is discrete rather than continuous, as we believe that the former framework is more suitable to represent the sequence of actions and decisions which lead to the formation of the population shares. Namely, in view of embracing a new preference structure, agents need to perform the consumption activity, to evaluate the satisfaction degree resulting from their previous choice, to gather information on the other structure of preferences and to compare the various satisfaction levels, in order to make their next choice. The modeling representation of those actions and decisions requires a time structure which considers an interval between two consecutive time instants. In our discrete-time framework, we obtained, in addition to the static coexistence phenomena between groups detected in $[1,2]$, also dynamical coexistence phenomena, oscillatory in nature, in which agents' choices change over time and are not confined just on steady states, describing stationary choices. In fact, everyday life observation, supported by empirical data, highlights erratic behaviors in agents' choices, and in particular oscillatory consumption phenomena, such as the fashion cycle (see, e.g., $[3,4])$, not reproduced by $[1,2]$.

In this respect, we remark that the setting here proposed could be modified in order to make it suitable to describe the fashion cycle. To such aim, more structure should be added to our model, admitting the possibility of different consumer lifestyles, that is, of certain preferences configurations over the various consumption goods, and introducing variables describing the attractiveness of each lifestyle.

Another possible line of research would concern the application of the methodology used in the present paper to contexts with strategic interaction, similar, for instance, to those considered in $[19,20]$, which share with our work the focus on the possible eventual coexistence among heterogeneous agents, as well as the evolutionary approach. Assuming strategic complementarity and/or substitutability, we could analyze the possible dynamical effects produced by heterogeneity between groups in those settings, too.

\section{Conflicts of Interest}

The authors declare that there are no conflicts of interest regarding the publication of this article.

\section{References}

[1] J. Chang and R. Stauber, "Evolution of preferences in an exchange economy," Economics Letters, vol. 103, no. 3, pp. 131$134,2009$. 
[2] A. Naimzada and M. Pireddu, "Endogenous evolution of heterogeneous consumers preferences: multistability and coexistence between groups," Economics Letters, vol. 142, pp. 22-26, 2016.

[3] P. E. Earl, Lifestyle Economics: Consumer Behaviour in a Turbulent World, Wheatsheaf Books, Brighton, England, 1986.

[4] D. E. Robinson, "Style changes: Cyclical, inexorable, and foreseeable," Harvard Business Review, vol. 53, pp. 121-131, 1975.

[5] G. Ponthiere, "Existence and stability of overconsumption equilibria," Economic Modelling, vol. 28, no. 1-2, pp. 74-90, 2011.

[6] W. H. Sandholm, Population Games and Evolutionary Dynamics, The MIT Press, Cambridge, Mass, USA, 2010.

[7] P. D. Taylor and L. B. Jonker, "Evolutionarily stable strategies and game dynamics," Mathematical Biosciences, vol. 40, no. 1-2, pp. $145-156,1978$.

[8] A. Bisin and T. Verdier, "The economics of cultural transmission and the dynamics of preferences," Journal of Economic Theory, vol. 97, no. 2, pp. 298-319, 2001.

[9] L. E. Blume and D. Easley, "Optimality and natural selection in markets," Journal of Economic Theory, vol. 107, no. 1, pp. 95-135, 2002.

[10] F. Cavalli, A. Naimzada, and M. Pireddu, "A family of models for Schelling binary choices," Physica A: Statistical Mechanics and its Applications, vol. 444, pp. 276-296, 2016.

[11] P. Dindo, "A tractable evolutionary model for the minority game with asymmetric payoffs," Physica A: Statistical Mechanics and its Applications, vol. 355, no. 1, pp. 110-118, 2005.

[12] C. Hommes, Behavioral Rationality and Heterogeneous Expectations in Complex Economic Systems, Cambridge University Press, Cambridge, UK, 2013.

[13] S. Wiggins, Introduction to Applied Nonlinear Dynamical Systems and Chaos, vol. 2, Springer, New York, NY, USA, 2003.

[14] B.-S. Du, "Point bifurcations for some one-parameter families of interval maps," Bulletin of the Institute of Mathematics, Academia Sinica, vol. 21, no. 3, pp. 187-202, 1993.

[15] B.-S. Du, "Point bifurcations and bubbles for some oneparameter families of quadratic polynomials," Bulletin of the Institute of Mathematics, Academia Sinica, vol. 25, no. 1, pp. 1-9, 1997.

[16] C. H. Hommes, "Adaptive learning and roads to chaos: the case of the cobweb," Economics Letters, vol. 36, no. 2, pp. 127-132, 1991.

[17] C. H. Hommes, "Dynamics of the cobweb model with adaptive expectations and nonlinear supply and demand," Journal of Economic Behavior \& Organization, vol. 24, no. 3, pp. 315-335, 1994.

[18] M.-C. Li, "Point bifurcations and bubbles for a cubic family," Journal of Difference Equations and Applications, vol. 9, no. 6, pp. 553-558, 2003.

[19] A. Heifetz, C. Shannon, and Y. Spiegel, "The dynamic evolution of preferences," Economic Theory, vol. 32, no. 2, pp. 251-286, 2007.

[20] A. Heifetz, C. Shannon, and Y. Spiegel, "What to maximize if you must," Journal of Economic Theory, vol. 133, no. 1, pp. 31-57, 2007. 


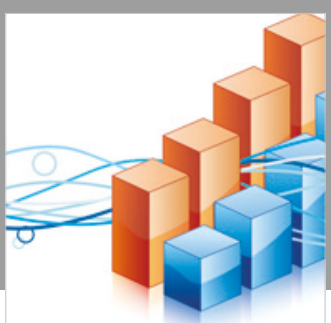

Advances in

Operations Research

\section{-n-m}
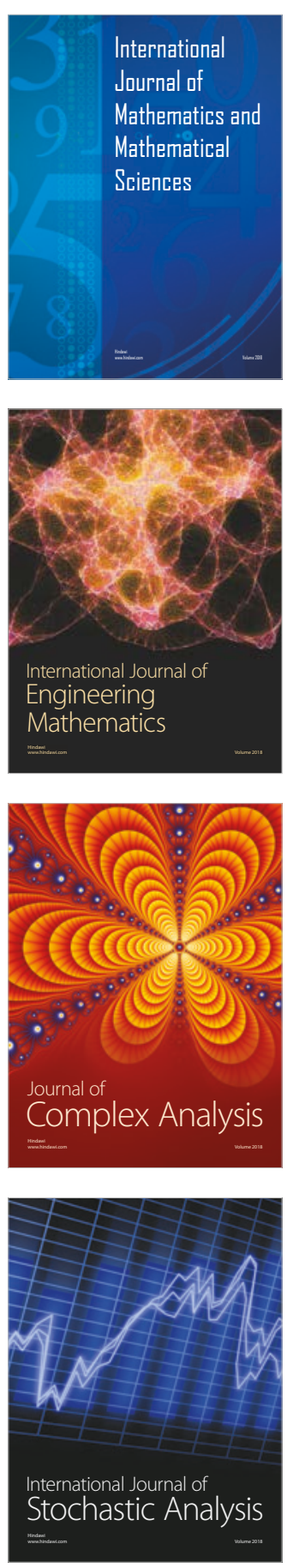
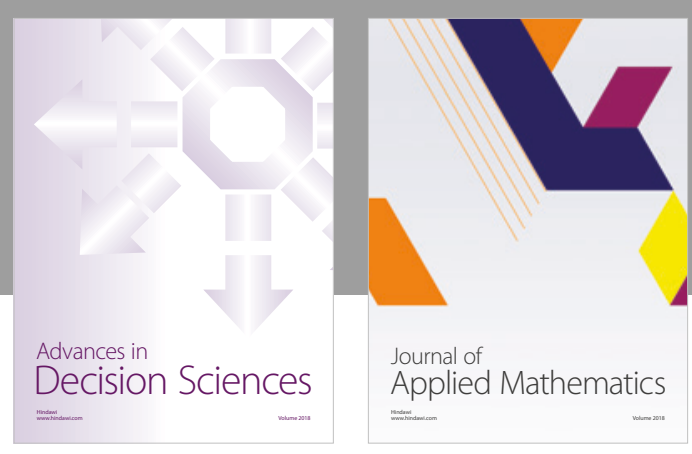

Journal of

Applied Mathematics
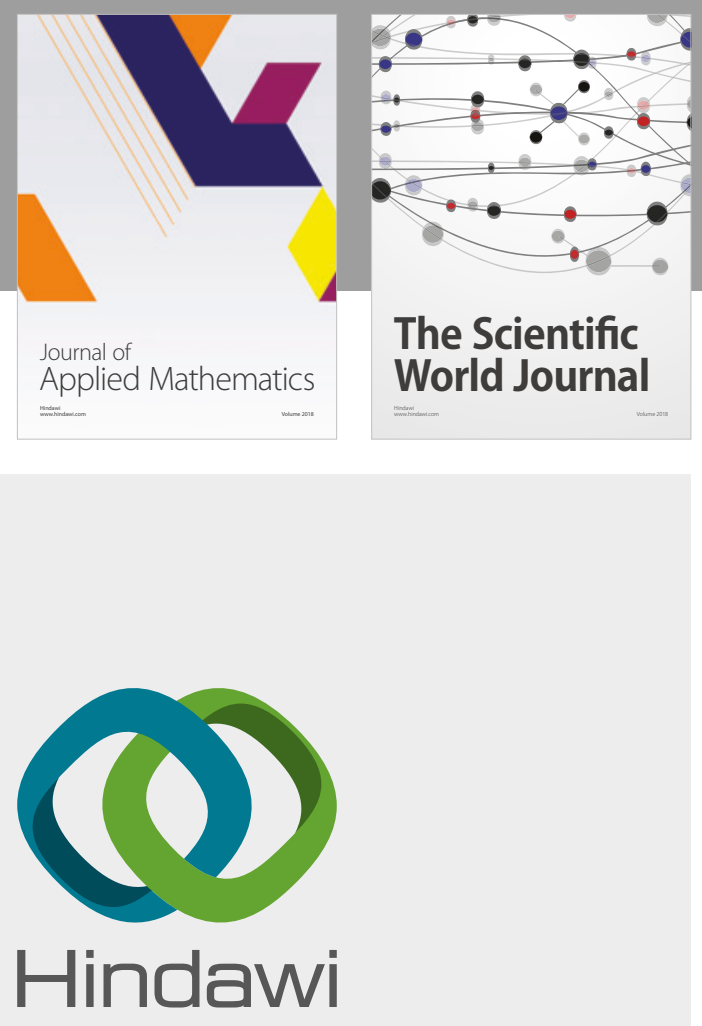

Submit your manuscripts at

www.hindawi.com

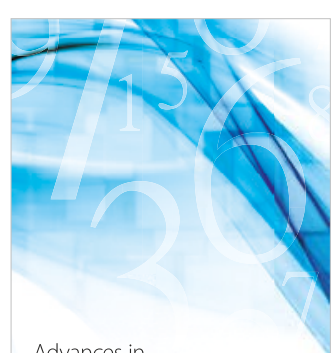

Advances in
Numerical Analysis
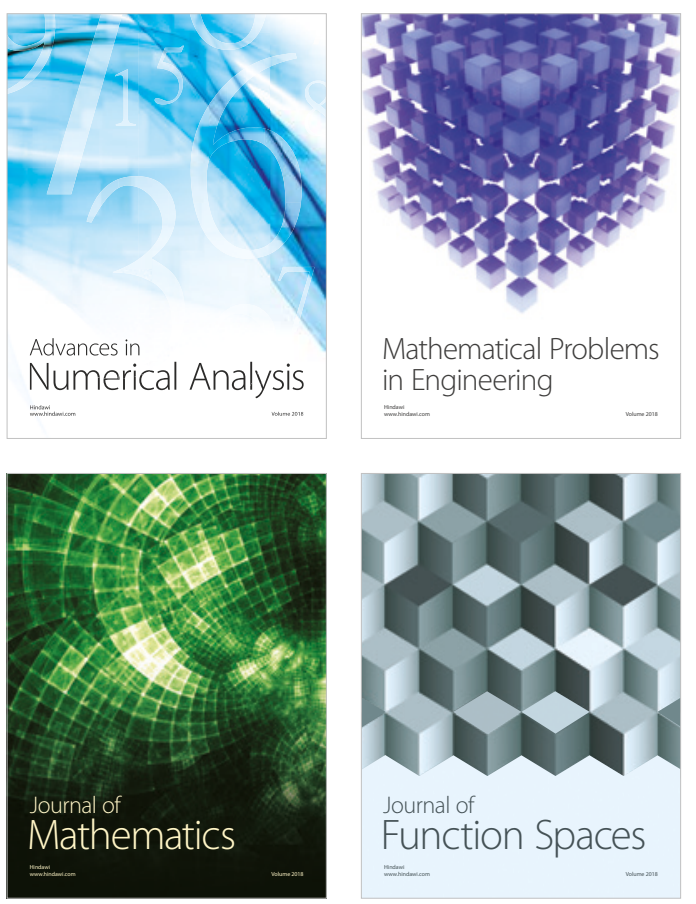

Mathematical Problems in Engineering

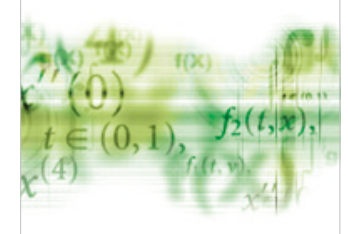

International Journal of

Differential Equations

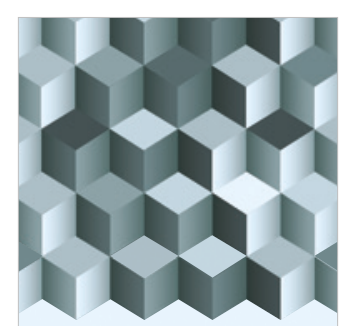

Journal of

Function Spaces

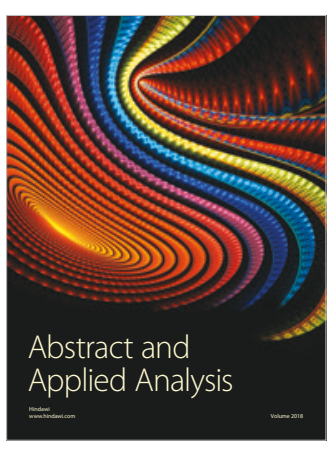

The Scientific

World Journal

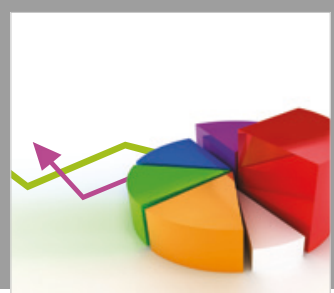

Journal of

Probability and Statistics
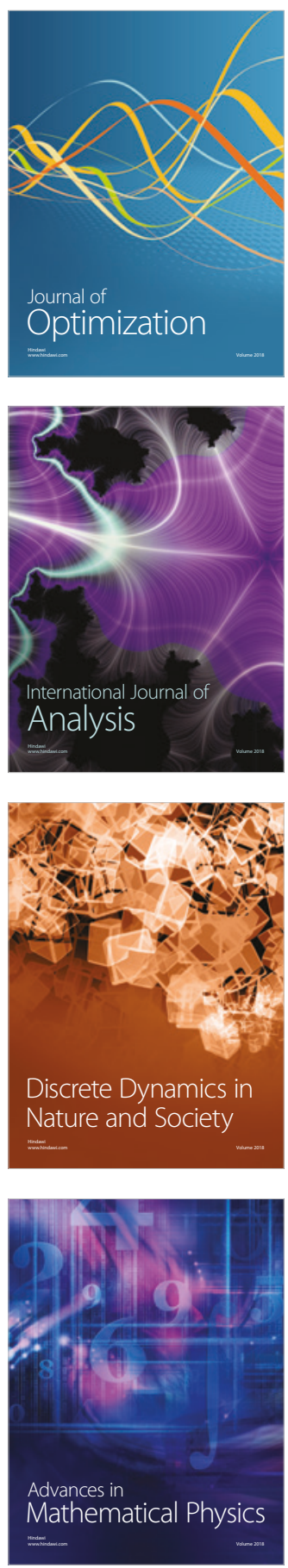